\title{
Effective of Behavioral Treatment Combined with Psychological Counseling for Female Coital Disorder of Chinese
}

\author{
Shu-Qi Ge1, Xin Tao ${ }^{*}$ \\ ${ }^{1}$ Department of Infertility and Sexual Medicine, the Third Affiliated Hospital, Sun-Yet Sen University, Guangzhou, China \\ ${ }^{2}$ Center for Reproductive Medicine, the Third Affiliated Hospital, Sun-Yet Sen University, Guangzhou, China \\ Email: *doctort@163.com
}

How to cite this paper: Ge, S.-Q. and Tao, X. (2017) Effective of Behavioral Treatment Combined with Psychological Counseling for Female Coital Disorder of Chinese. Health, 9, 1509-1515.

https://doi.org/10.4236/health.2017.911111

Received: September 9, 2017

Accepted: October 22, 2017

Published: October 25, 2017

Copyright @ 2017 by authors and Scientific Research Publishing Inc. This work is licensed under the Creative Commons Attribution International License (CC BY 4.0).

http://creativecommons.org/licenses/by/4.0/

\section{c) (i) Open Access}

\begin{abstract}
Objective: To explore the effects of the behavioral treatment combined with psychological counseling on female coital disorder in China. Methods: One hundred and sixteen female patients with coital disorder were divided into sexual behavior training combined with psychological counseling group (group A, 59 cases) and psychological counseling alone group (group B, 57 cases). Results: Forty-five cases cured (76.27\%), eight eases improved (13.56\%), and six cases invalid (10.17\%), the general rate of effectiveness being $89.83 \%$ in group A while eleven cases cured (19.30\%), twenty-one cases improved (36.84\%), and twenty-five cases invalid (43.86\%), the general rate of effectiveness being $56.14 \%$ in group $B$. The curative effect of group A was better than group B $(P<0.05)$. Conclusions: The behavioral treatment combined with psychological counseling is significantly effective in treating female coital disorder than psychological counseling alone and its long term follow-up results show that most of patients were satisfied with their sex life. However, the response of behavioral treatment of some patients who had bad experience on sexual things in the progress of their growing up or had long course of disease were not so good which deserves our special attention in the future study.
\end{abstract}

\section{Keywords}

Female Sexual Dysfunction, Female Coital Disorder, Behavioral Treatment

\section{Introduction}

Female coital disorder also known as female sexual intercourse difficulties, refers to that the vagina cannot tolerate the erection of the penis inserted or vaginal 
loss, resulting in female sexual intercourse unsuccessful or that sexual intercourse is very pain so that sexual intercourse is difficult to complete. Female sexual dysfunction is a common disease that affects the quality of life of women [1]. There are few reports about Chinese women with female coital disorder.

In clinical work, we found that there are some patients eliminating genital abnormalities and male factors, although they have sexual desire and repeated attempts still cannot have successful sexual life, which seriously affected the husband and wife life, the marriage stability and the well-being of family. If improper handling may cause a loss of libido and sexual arousal disorder, orgasmic dysfunction and other sexual dysfunction, etc. In the clinical treatment of female coital disorder, there is still no standard treatment in our country as well as abroad [2]. In this study, we focused on the efficacy and evaluation of sexual behavior training combined with psychological counseling or psychological counseling alone for such female coital disorder of one hundred and sixteen women in the past ten years in our department.

\section{Materials and Methods}

\subsection{Source and Grouping}

Choose 116 cases of adult women due to sexual intercourse obstruction from February 2006 to February 2016 in our department. The inclusion criteria refer to the American Psychiatric Association for Classification of Sexual Disorders [3] (DSM-V), that the patients eliminate genital abnormalities and male factors, although have sexual desire and repeated attempts still cannot have successful sexual life were included in our study. Specific to our cases, mainly based on whether can successful sexual intercourse be included in the diagnosis of sexual intercourse disorders. The examination of the internal and external genital organs and blood examination basic sex hormones were no abnormalities, while exclude those suffering from organic disorders which affecting normal sexual intercourse such as diabetes, cardiovascular and thyroid dysfunction, endometriosis, pelvic trauma, reproductive tract inflammation, sexually transmitted diseases and eliminate the male erectile dysfunction (including physical and mental aspects) or other factors affecting the sexual intercourse of the man.

Were randomly divided into sexual behavior training combined with psychological counseling group (group A, 59 cases) and psychological counseling alone group (group B, 57 cases). There was no significant difference between the two groups in terms of age, occupation distribution, education level, marital status, growth environment and sexual knowledge. The general information between the two groups is shown in Table 1.

\subsection{Methods}

The general situation of the subjects was recorded and analyzed in detail: the general situation of the subjects included age, course of disease, educational 
Table 1. Comparison of two groups of general information.

\begin{tabular}{|c|c|c|c|c|c|}
\hline & & & $\begin{array}{l}\text { Group A } \\
\text { (59 cases) }\end{array}$ & $\begin{array}{l}\text { Group B } \\
\text { (57 cases) }\end{array}$ & $P$ \\
\hline \multirow{16}{*}{$\begin{array}{l}\text { The basic } \\
\text { condition } \\
\text { of the patient }\end{array}$} & $\begin{array}{l}\text { Age } \\
\text { (years) }\end{array}$ & & $29.63 \pm 3.17$ & $28.70 \pm 3.27$ & $>0.05$ \\
\hline & Occupation distribution & Staff & $54.24 \%$ & $52.63 \%$ & $>0.05$ \\
\hline & & Manager & $6.78 \%$ & $7.02 \%$ & \\
\hline & & Teacher & $10.17 \%$ & $17.54 \%$ & \\
\hline & & Business service provider & $18.64 \%$ & $15.79 \%$ & \\
\hline & & Freelancer & $3.39 \%$ & $5.51 \%$ & \\
\hline & & Technical personnel & $5.08 \%$ & $3.51 \%$ & \\
\hline & & Unemployed & $1.69 \%$ & 0 & \\
\hline & & Primary and secondary school education & $6.78 \%$ & $12.28 \%$ & \\
\hline & Education level distribution & $\begin{array}{l}\text { Technical secondary school education } \\
\text { or Junior college degree }\end{array}$ & $37.29 \%$ & $26.32 \%$ & $>0.05$ \\
\hline & & Bachelor degree or above & $55.93 \%$ & $61.40 \%$ & \\
\hline & & Married & $86.44 \%$ & $91.22 \%$ & \\
\hline & Marital status & Unmarried & $11.86 \%$ & $7.02 \%$ & $>0.05$ \\
\hline & & Cohabitation & $1.69 \%$ & $1.75 \%$ & \\
\hline & Course of disease(month) & & $26.58 \pm 24.94$ & $24.96 \pm 24.40$ & $>0.05$ \\
\hline & Treatment times & & $3.88 \pm 1.55$ & $3.43 \pm 1.25$ & $>0.05$ \\
\hline \multirow{5}{*}{$\begin{array}{l}\text { Growth } \\
\text { environment }\end{array}$} & $\begin{array}{l}\text { Enlightened parents, harmonious } \\
\text { relationship between parents }\end{array}$ & & $20.34 \%$ & $17.54 \%$ & $>0.05$ \\
\hline & Family education strict and traditional & & $57.63 \%$ & $57.89 \%$ & \\
\hline & $\begin{array}{l}\text { Parents emotional discord or } \\
\text { separated for a long time }\end{array}$ & & $11.86 \%$ & $14.04 \%$ & \\
\hline & $\begin{array}{l}\text { Having a bad sexual experience } \\
\text { at an early age }\end{array}$ & & $8.47 \%$ & $7.02 \%$ & \\
\hline & Have emotional trauma & & $1.69 \%$ & $3.51 \%$ & \\
\hline \multirow{5}{*}{ The relationship } & Conjugal love & & $79.66 \%$ & $78.95 \%$ & $>0.05$ \\
\hline & Flat feelings & & $13.54 \%$ & $12.28 \%$ & \\
\hline & Emotional coldness & & $3.39 \%$ & $5.26 \%$ & \\
\hline & Breaking up & & $1.69 \%$ & $1.75 \%$ & \\
\hline & Divorced & & $1.69 \%$ & $1.75 \%$ & \\
\hline
\end{tabular}

level, economic income, marital status, menstrual history, BMI, waist circumference, blood pressure, past medical history, history of surgery and medication history. With a comprehensive understanding of the history of medication and psychological history, if the patient has a history of sexual abuse stated, is to learn more about when patients recovering from sexual abuse (with or without treatment) in the past, is there a history of recurrent depression, history of drug 
abuse, self-injury or a history of debauchery, whether patients can't trust others, especially those who hurt her, whether exaggerated anxiety drug demand or need to please demand and can't refuse.

Therapy: 1) sexual behavior training combined with psychological counseling group (group A): Mainly conduct the mold insertion training, reduce vulva sensitivity, according to the degree of patient acceptance, training from small-medium-large sized mold were conduct by sexual behavior therapist in our department, until the penis can be successful inserted into the vagina for sexual life. At the same time supplemented by explaining reproductive anatomical knowledge and psychological counseling, to eliminate the fear of sexual intercourse, adjust the mentality and learn sexual intercourse skills. Each treatment requires participation with her husband. At the end of each treatment, the corresponding homework was arranged according to the progress of each patient, told them conducting the mold insertion training by finger or mold or gradually try to intercourse at home; 2) psychological counseling alone group (group B): explain the knowledge of reproductive anatomy, give psychological counseling, to eliminate the fear of sexual intercourse, psychological adjustment, learning sexual intercourse skills.

Evaluation criteria: efficacy evaluation criteria: clinical symptoms disappeared, Patients and spouses were satisfied is cured. Clinical symptoms were relieved, patients and spouses were basically satisfied, without further treatment for the better is improved. The treatment was once a week and the treatment time was one hour each time. After treatment for more than ten times, the main symptoms no significant improvement, the patient has been reluctant to continue treatment is invalid.

\subsection{Statistical Method}

Adopt SPSS 13.0 statistical software for statistical processing, measurement data was described by the mean \pm standard deviation $(\bar{X} \pm S)$. The comparison between the two groups was made by the normality test and the homogeneity of variance test, Kamo Sai used the two independent sample $t$ test, and the $t$ test was used when the variance was not uniform. Nonparametric tests were used for variables that do not conform to the normal distribution. The rank sum test of ordinal variable data was used to compare the efficacy between the two groups. According to the test level of alpha $=0.05, P<0.05$ was statistically significant.

\section{Result}

1) The general information between the two groups is shown in Table 1.

2) Comparison of clinical efficacy between the two groups after treatment is shown in Table 2.

\section{Discussion}

Female sexual dysfunction (FSD) is a common disease affecting the quality of life 
Table 2. Comparison of clinical efficacy between the two groups after treatment.

\begin{tabular}{ccccccc}
\hline Grouping & Cases & Cure & Improvement & Invalid & Total effective rate & $P$ \\
\hline Group A & 59 & $45(76.27 \%)$ & $8(13.56 \%)$ & $6(10.17 \%)$ & $89.83 \%$ & $<0.01$ \\
Group B & 57 & $11(19.30 \%)$ & $21(36.84 \%)$ & $25(43.86 \%)$ & $56.14 \%$ & $<0.01$ \\
\hline
\end{tabular}

of women, with an incidence of about $20 \% \sim 50 \%$ [2] [3] [4]. Female coital disorder also known as female sexual intercourse difficulties, refers to that the vagina cannot tolerate the erection of the penis insertion or vaginal loss, the penis tries or completely into the vagina and (or) when the penis in the vagina friction the women feel persistent or recurrent pain, resulting in the woman's unsuccessful sexual intercourse or sexual intercourse is very painful so that sexual intercourse is difficult to complete, which is a kind of FSD disease. For a long time, the women's sexual health did not get enough attention, especially in our country, due to the influence of traditional culture, women's sexual dysfunction is seriously ignored, and female sexual dysfunction is precisely a very important issue. Foreign data [5] shows that female sexual dysfunction, and the rising divorce rate, family violence, a single mother, such phenomena are closely related, which seriously affects the relationship between husband and wife, family harmony and the quality of life.

In this study, the patients' basic hormone levels were normal, genital examination without anatomical problems, with normal menstrual cycle and normal libido, with normal sexual desire, and the penis erectile function of male is normal, both husband and wife sexual orientation were normal. However, the penis was not successfully inserted into the vagina by repeated attempts and can't succeed to start having sex. Analysis of the reason found that patients tend to exist sexual intercourse difficulties, fear, avoidance, and high muscle tension when partners trying put penis into the vagina.

This study found that women with sexual intercourse disorder factors, due to the patient's fear of pain, lack of knowledge of both sides, strict family education, lack of sexual communication between the two sides, and the patient's own factors such as cleanliness, these are the reasons why sexual intercourse disorder occurs [6]. Deeply analyzing these causes, we emphasize that the treatment of sexual concepts and basic knowledge, such as sexual organs dirty, sexual fantasies and masturbation immoral or harmful and so on are ubiquitous problems in our country. Therefore, through the conversation and VCD teaching film, we carry out the necessary education, the basic establishment of a healthy sexual concept and sexual knowledge (including sexual skills), and then through the analysis, we give targeted psychological counseling. The existence of couples emotional problems, obvious depression and gynecological inflammation of the patient must first be treated until the situation improved to enter the treatment of behavioral therapy, such patients often has a long history of unsuccessful sexual intercourse which causes the emergence of husband and wife emotional problems, depression and other mental state, and even gynecological inflamma- 
tion, and when these problems arise, it often means that the condition is more serious, similar to the vicious cycle being entered. Focus on patients with mold insert training, requiring patients and spouses to actively cooperate with the treatment to gradually relieve the fear of sexual intercourse, and gradually understand the content of sexual intercourse to help patients establish a correct early sexual experience, and then enjoy the fun of sex, to prevent the occurrence of female sexual dysfunction. These treatments have achieved good therapeutic results.

However, there are still six cases of treatment in group A with ineffective and group B of twenty-five patients with treatment ineffective. Analysis of the reason found that the cases had bad sexual experience in the childhood with poor curative effect, and found that the duration of the disease effect on the treatment effect. The course of this group of patients within five years of treatment is satisfactory, but with the extension of the course of treatment, treatment effect gradually deteriorated. In the treatment of failure of six cases in group A, one case is because the course is too long, up to ten years. Although therapists try their best, the patient is more willing to actively cooperate, but always unable to overcome the obstacles in the patient's mind and considers that the possible long-term illness has been secondary cause of more serious psychological barriers and simple behavior training cannot overcome the problem which need a professional psychologist to help solve the problem. There are five cases of people have had a poor sexual experience in childhood of Group A, of which four women treatment effect is always poor. There are four women had poor sexual experience in childhood of group B, of which two people treatment is poor. It is suggested that timely and effective childhood sexual education and sexual protection for the growth process is essential and even affects the adult sexual attitude, seriously affecting the normal life.

Clinical findings that, the length of the course of a direct impact on the treatment effect (the longer the course, the worse the treatment effect, such as: more than 1 year course, treatment significantly increased; $<1$ year course, the effect of treatment was better than more than 1 year, but still better than individual psychological counseling effect).

We found that in this type of female sexual intercourse disorder disease, the lack of sexual knowledge, knowing nothing about sex, fear; sexually assaulted in the process of growing up and lack of sexual communication for various reasons were important psychogenic risk factors. Sexual abuse for various reasons by the growing lack of exchanges and other psychological factors were important risk factors. For these cases with too long course of disease and poor therapeutic effects, there will be the key point and difficulty of treatment in our future research. In terms of treatment, explaining sexual knowledge and psychological counseling at the same time combined with sexual behavior training in women with female sexual intercourse disorders, the effect was significantly better than psychological counseling alone treatment $(P<0.01)$. It shows that the disease is 
psychosomatic disease, and only psychological counseling combined with sexual behavior training can achieve the obvious effect. Although our study is very novel and effective, but still have some deficiencies. Because the incidence of such diseases is low and there are few reports at home and abroad, there are few articles we can refer to. We attempt to observe the effective treatment, but more cases and longer observation are needed. Next, we need more detailed study and analysis of the cause of the disease in order to refine the symptomatic treatment.

\section{References}

[1] Ben-Zion, I.Z., Shiber, A. (2004) Female Sexual Dysfunction (FSD). Harefuah, 143, 417-419, 462.

[2] Allahdadi, K.J., Tostes, R.C.A. and Webb, R.C. (2009) Female Sexual Dysfunction: Therapeutic Options and Experimental Challenges. Cardiovascular \& Hematological Agents in Medicinal Chemistry, 7, 260-269. https://doi.org/10.2174/187152509789541882

[3] Binik, Y.M., et al. (2010) Response of the DSM-V Sexual Dysfunctions Subworkgroup to Commentaries Published in JSM. The Journal of Sexual Medicine, 7, 2382-2387. https://doi.org/10.1111/j.1743-6109.2010.01899.x

[4] Rasanen, M. (2003) Female Sexual Dysfunction. Duodecim, 119, 219-227.

[5] Aslan, E. and Fynes, M. (2008) Female Sexual Dysfunction. International Urogynecology Journal, 19, 293-305. https://doi.org/10.1007/s00192-007-0436-3

[6] Tao, X., Chen, J., Zhang, B., Zhang, E., Qi, T. and Cao, M. (2014) How Do the Chinese Make Love? A Community Based Survey in China. Health, 6, 1004-1011.

https://doi.org/10.4236/health.2014.610126 